\title{
Activity-dependent Fluorescent Staining and Destaining of Living Vertebrate Motor Nerve Terminals
}

\author{
William J. Betz, ${ }^{1}$ Fei Mao, ${ }^{2}$ and Guy S. Bewick ${ }^{1 a}$ \\ 'Department of Physiology, University of Colorado School of Medicine, Denver, Colorado 80262 and ${ }^{2}$ Molecular Probes, \\ Inc., Eugene, Oregon 97402
}

\begin{abstract}
Living motor nerve terminals from several species can be stained in an activity-dependent fashion by certain styryl dyes, such as RH414, RH795, and a new dye, FM1-43, which can be imaged independently of the others. The dyes evidently become trapped within recycled synaptic vesicles. In frog cutaneus pectoris muscle, bright fluorescent spots spaced regularly along the length of the nerve terminals appear after stimulation in the presence of the dye. The spots align well with postsynaptic ACh receptors and are persistent for many hours, unless further stimulation is given, in which case the spots disappear. Destaining, like staining, requires transmitter release and proceeds gradually over several minutes at high stimulus frequencies (e.g., $30 \mathrm{~Hz}$ ), and fluorescent spots in the same terminal disappear at about the same rate. We suggest that each spot is a cluster of hundreds of synaptic vesicles and that the mechanism of staining involves the ability of the dyes to partition reversibly into the outer leaflet of surface membranes, without being able to penetrate the entire membrane thickness. Then, during endocytosis following transmitter release, dye molecules become trapped in recycled synaptic vesicle membranes. The dyes therefore make it possible optically to study vesicle exocytosis and recycling in living nerve terminals in real time, and should be useful for marking terminals in a variety of preparations according to their level of activity.
\end{abstract}

Markers that label neurons in an activity-dependent fashion have been useful tools in neurobiology. HRP was the first such marker identified (Graham and Karnovsky, 1966); it is endocytosed by nerve terminals following nerve activity and marks recycled synaptic vesicles in fixed or frozen preparations (Holtzman et al., 1971; Ceccarelli et al., 1972, 1973; Heuser and Reese, 1973; reviewed in Zimmermann, 1979). Several hydrophilic

Received July 16, 1991; revised Sept. 13, 1991; accepted Sept. 18, 1991.

Mr. Steven Fadul provided unfailing technical assistance in all phases of this work. We thank Drs. Bruce Wallace and Paul Fuchs for many helpful discussions and for suggestions on manuscript drafts; Drs. A. R. Martin, Warren Wickelgren, Michael Browning, and Kathryn Howell (all University of Colorado Mcdical School), C. P. Ko (University of Southern California), and Antony Ridge (University of Bristol) for helpful advice; Dr. Vic Spitzer for generous equipment loans; Dr. Yu Li Wang (Worcester Institute) for his software gift; and Ms. Janet Lieber for expert electron microscopical assistance. This work was supported by research grants from MDA and NIH to W.J.B.

Correspondence should be addressed to Dr. William J. Betz, Department of Physiology/C-240, University of Colorado School of Medicine, Denver, CO 80220.

${ }^{a}$ Present address: Muscular Dystrophy Research Laboratories, Newcastle General Hospital, Westgate Road, Newcastle-Upon-Tyne, NE4 6BE, United Kingdom.

Copyright (c) 1992 Society for Neuroscience $0270-6474 / 92 / 120363-13 \$ 05.00 / 0$ fluorescent dyes have been shown to stain living motor nerve terminals of the garter snake in an activity-dependent fashion (Lichtman et al., 1985; Lichtman and Wilkinson, 1987). Unfortunately, these dyes, for unknown reasons, do not work well in other, more commonly used preparations, and so we sought new fluorophores that would stain a broader range of living preparations in a nontoxic, activity-dependent fashion. The styryl dyes RH414, RH795, and a new derivative of RH414, FM143 (which emits at a shorter wavelength), fulfill these criteria, staining frog, rat, and mouse motor nerve terminals with an intensity that depends upon the amount of transmitter released (preliminary account in Bctz and Bcwick, 1990). In addition, stained terminals can be destained in dye-free medium, and destaining also depends upon transmitter release. Several observations suggest that the dyes mark membranes of recycled synaptic vesicles. The dyes may therefore be useful tools for optical studies of synaptic vesicles exocytosis and recycling, and for marking living terminals in a broad range of preparations according to their level of functional activity.

\section{Materials and Methods}

Staining. Experiments were performed using Rana pipiens cutaneous pectoris nerve-muscle preparations between October and April. Preparations were dissected and pinned in a Sylgard-lined chamber, exposed to dye (RH414, 10-40 $\mu \mathrm{M}$; FM1-43, 1-2.5 $\mu \mathrm{M}$ ) dissolved in normal frog Ringer's (mM: $\mathrm{NaCl}, 115 ; \mathrm{KCl}, 2 ; \mathrm{CaCl}_{2}, 1.8 ; \mathrm{NaHCO}_{3}, 2.4$ ) for 1-15 min, and then washed in normal Ringer's. Nerve terminals were stimulated either by electrical stimulation of the muscle nerve with a suction electrode [typically $1 \mathrm{~Hz}$ continuous stimulation for $10-15 \mathrm{~min}$ with 2 sec trains $(30-50 \mathrm{~Hz})$ every $30 \mathrm{sec}$ ) or by exposure for $1-5 \mathrm{~min}$ to dye dissolved in high-[K+ $\left.\mathrm{K}^{+}\right]$Ringer's (usually $60 \mathrm{~mm}$; external $\left[\mathrm{Na}^{+}\right]$was reduced by an equivalent amount). Preparations were washed for 5$120 \mathrm{~min}$ before viewing.

FM1-43 [ $N$-(3-(triethyl ammonium) propyl)-4-(4-dibutylaminostyryl pyridinium, dibromide; see Fig. $13 \mathrm{~A}$ ] is similar to RH414 but contains only one double bond (and so emits at a shorter wavelength). We studied spectral properties of both dyes and $f($ und that, in preparations stained with both dyes, FM1-43 could be inraged independently in the green (450-550 nm emission filter), and RH414 could be imaged almost, but not completely, independently in the red $(600-700 \mathrm{~nm}$ emission filter). FM1-43 also has a longer hydrophobic tail than does RH414 and so dissolves in membranes more easily, requiring a shorter incubation time and lower concentration than RH414. The RH dycs were developed as membrane potential-sensitive fluorophores (Grinvald et al., 1982, 1988), but in the present experiments potential-dependent changes in fluorescence would be, at most, less than a few percent and so would not significantly affect our conclusions. In addition, we found that the dyes can block postsynaptic acetylcholine receptors (AChRs), an action that washes out easily, and thus did not affect the destaining experiments described here. A detailed account of this effect is in preparation.

For labeling AChRs and nerve terminals simultaneously, preparations were exposed to $3.75 \mu \mathrm{g} / \mathrm{ml}$ rhodamine-conjugated $\alpha$-bungarotoxin (Molecular Probes, Inc.) in normal Ringer's for $20 \mathrm{~min}$, washed and 


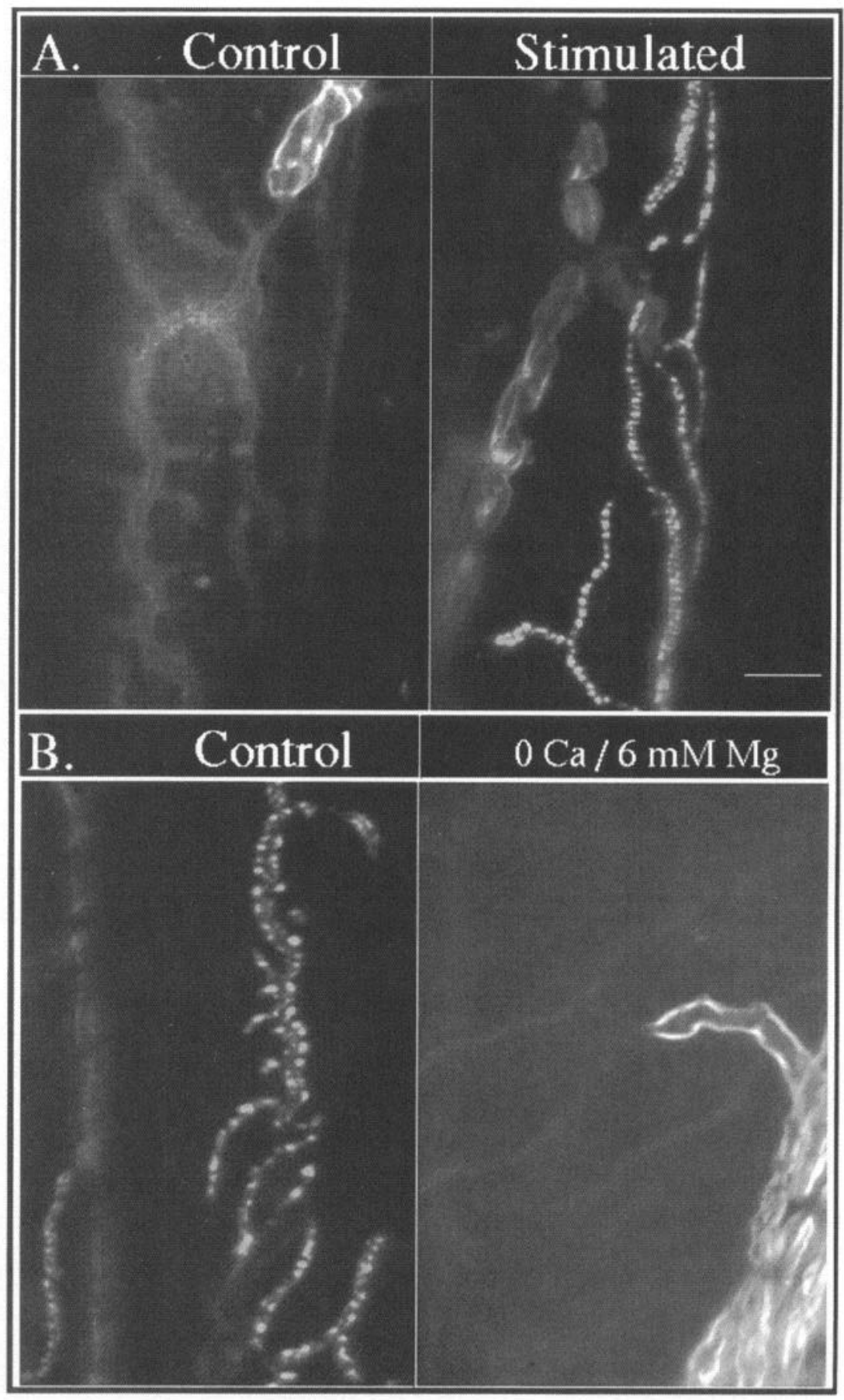

Figure 1. Staining of frog motor nerve terminals with $\mathrm{RH} 414$ depended upon transmitter release. $A$, In the control preparation (left), which received no nerve stimulation during exposure to dye, myelin stained brightly, but nerve terminals are only faintly visible. In the stimulated preparation (right) bright beadlike fluorescent spots appeared in the nerve terminals. The nerve was electrically stimulated ( $5 \mathrm{sec}$ trains of $10-20 \mathrm{~Hz}$ every $30 \mathrm{sec}$ for $15 \mathrm{~min}$ superimposed on continuous $1 \mathrm{~Hz}$ stimulation). $B$, Staining depended on transmitter release. These preparations were stimulated by exposure to $60 \mathrm{mM} \mathrm{K}^{+}$Ringer's $(2 \mathrm{~min})$, rather than by electrical stimulation of the nerve. Left, Stimulation in normal $\left[\mathrm{Ca}^{2+}\right] /\left[\mathrm{Mg}^{2+}\right]$. Right, Stimulation in $\mathrm{Ca}^{2+}$-free Ringer's (with $6 \mathrm{~mm} \mathrm{Mg}^{2+}$ added) blocked nerve terminal, but not myelin staining. Scale bar, $10 \mu \mathrm{m}$.

exposed to $1 \mu \mathrm{M}$ FM1-43 in $60 \mathrm{mM} \mathrm{K}^{+}$-Ringer's for $2 \mathrm{~min}$, and then washed for $15 \mathrm{~min}$.

Electron microscopy. Freshly dissected muscles were stained with 2 $\mu \mathrm{M}$ FM1-43 in $60 \mathrm{~mm}\left[\mathrm{~K}^{+}\right]$for $5 \mathrm{~min}$ and washed, and terminals were imaged under the light microscope to confirm that staining had occurred. Controls were normal, unstained contralateral muscles. The muscles were then prepared for conventional electron microscopy. They were fixed in $2.5 \%$ glutaraldehyde for $1 \mathrm{hr}$, postfixed in $1 \% \mathrm{OsO}_{4}$ for $1 \mathrm{hr}$, dehydrated, embedded in Epon, and sectioned. Sections were stained with $5 \%$ uranyl acetate in $70 \%$ methanol for 10 min, placed in Reynold's lead citrate for $5 \mathrm{~min}$, and viewed with a Zeiss $9 \mathrm{~S}-2$ electron microscope. Only terminals on surface muscle fibers were studied.

Fluorescence microscopy. The preparation was viewed with a Leitz Laborlux epifluorescence microscope with a $100 \mathrm{~W} \mathrm{Hg}$ lamp and 1$10 \%$ (usually $2 \%$ ) neutral density transmission filters. Objective lenses used were Zeiss $40 \times$ water immersion ( $0.75 \mathrm{NA})$, Leitz $100 \times$ oil immersion (1.32 NA), and Zeiss $63 \times$ oil immersion (1.4 NA). A coverslip fragment was glued to the oil immersion objectives. Excitation and emission filters in the Leitz Ploemopaks were replaced with bandpass filters (Omega Optical, Inc.) in sliding holders. These consisted of four $10 \mathrm{~nm}$ bandpass excitation filters, each centered on a peak of the $\mathrm{Hg}$ lamp, and five $100 \mathrm{~nm}$ bandpass emission filters (center points from 500 to $700 \mathrm{~nm}$ in $50 \mathrm{~nm}$ increments). Thus, for FM1-43, we normally used a $430-440 \mathrm{~nm}$ excitation filter, a Leitz $\mathrm{H} 3$ dichroic mirror, and the 500-600 nm emission filter, and for rhodamine we used a 541-551 $\mathrm{nm}$ excitation filter, a Leitz N2 dichroic, and the 600-700 nm emission filter.

For FM1-43 in preparations also stained with rhodamine, we used filters as noted above except for a 450-550 nm (green) emission filter. Control observations of end plates stained with only one dye showed 


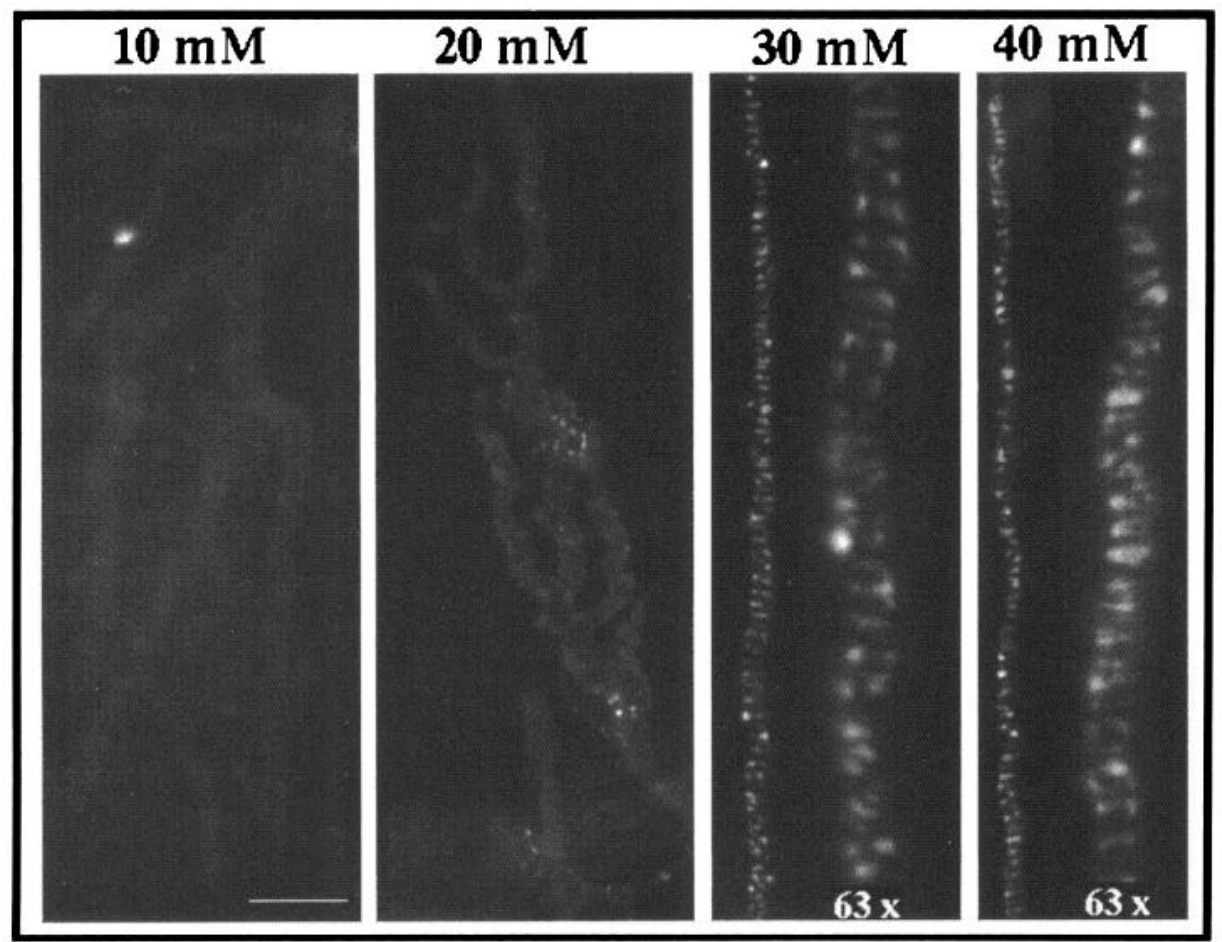

Figure 2. The intensity of staining varied with the amount of transmitter release. Muscles were exposed to $2 \mu \mathrm{M}$ FM1-43 for 5 min in high- $\mathrm{K}^{+}$Ringer's $\left(\mathrm{K}^{+}\right.$concentration is marked at the top of each panel). At $10 \mathrm{~mm}\left[\mathrm{~K}^{+}\right]$, little staining occurred. At $20 \mathrm{~mm}$, a few small spots were visible, especially over Schwann cell nuclei. At $30 \mathrm{~mm}$ and 40 $\mathrm{mm}$, the full staining pattern was produced; these terminals were imaged with both $40 \times($ left $)$ and $63 \times(r i g h t)$ objectives. Images at the same magnification were acquired and processed identically; the $63 \times$ images were digitally magnified by 1.6 . Scale bar, $10 \mu \mathrm{m}(4 \mu \mathrm{m}$ at $63 \times)$.

that spectral separation of the two dyes was virtually complete under these conditions; no "bleed through" was detectable. (The rhodamine $\alpha$-bungarotoxin signal was undetectable in the green. Under optimal conditions, the FM1-43 signal can be detected in the red, but the rhodamine signal was so strong in these experiments that the contribution from FM1-43 was negligible.)

Only terminals on surface muscle fibers were studied. Most often the terminals lay on the top surface of the muscle fiber and so were viewed "from above," as opposed to terminals that lay on the lateral margins of muscle fibers, which were viewed "from the side."

Image processing. Images were captured either with a Dage 66 SIT camera (1-2 sec averaging; with Imaging Technology 151 digitizing hardware) or Photometrics Star I chilled CCD camera (usually $3 \mathrm{sec}$ exposure; gain 4). Images were acquired, stored, and processed with a Silicon Graphics Personal Iris computer running software from G. W. Hannaway \& Associates and printed with a Kodak XL7700 printer. Digital processing involved only linear global intensity stretching or compression; differential spatial enhancements were not performed on images shown here. For destaining time course studies, 12 bit images were acquired with the Star I camera, pixel values were compressed identically to 8 bits, images were aligned, spots were marked on the first image, average intensities were computed for each spot in every image, and, for each picture, the averages of all measured spots were plotted (e.g., see Figs. 10,12). In some cases, areas of interest were enlarged $3 \times$ [with Gouraud (bilinear interpolation) averaging] and realigned; this made spot marking more accurate.

\section{Results}

\section{Staining required transmitter release}

As shown in Figure $1 A$ (left panel), after frog cutaneus pectoris muscles were incubated with dye in the absence of nerve stimulation the terminals were scarcely visible, although myelin stained brightly. This low level of background staining virtually disappeared after washing in dye-free medium for 30-60 min. If, however, the nerve was stimulated during dye exposure (e.g., $10 \mathrm{~Hz}$ for 3-5 min), bright fluorescent spots $1-2 \mu \mathrm{m}$ in diameter appeared along the length of nerve terminals (Fig. 1A, right panel), and this staining could not be washed out (see below). Further experiments, described below, led us to conclude that each spot consisted of a cluster of stained recycled synaptic vesicles.

Nerve terminal staining did not require action potentials; it could also be produced by exposure to elevated potassium concentrations in the presence of the dye (Fig. $1 B$, left side). Terminal staining did, however, require transmitter release; if transmitter release was blocked with low $\left[\mathrm{Ca}^{2+}\right] / \mathrm{high}\left[\mathrm{Mg}^{2+}\right]$ Ringer's during dye exposure, virtually no nerve terminal staining occurred (Fig. $1 B$, right side).

We studied a variety of stimulation paradigms to identify conditions for optimal staining. For example, as shown in Figure 2 , a 5 min exposure to $1 \mu \mathrm{M} \mathrm{FM} 1-43$ in $10 \mathrm{~mm}$ or $20 \mathrm{~mm}\left[\mathrm{~K}^{+}\right]$ produced little nerve terminal staining, while 30 or $40 \mathrm{~mm}\left[\mathrm{~K}^{+}\right]$ produced intense staining. Electrical stimulation of the nerve in the presence of dye also produced staining that varied with the amount of stimulation (results not shown). Typically, $5 \mathrm{sec}$ trains at $10-20 \mathrm{~Hz}$ every $30 \mathrm{sec}$ for $10-15 \mathrm{~min}$ in $1-2 \mu \mathrm{M}$ FM1-43 produced maximally intense staining.

In a few experiments, we tested the ability of the dyes to stain terminals after the stimulation period. The muscle nerve was stimulated electrically $(50 \mathrm{~Hz}$ for $1 \mathrm{~min})$ in the absence of dye, and the dye was applied immediately after the stimulation ended. These terminals developed fluorescent spots similar to, though generally not as bright as, those stimulated in the presence of dye. This suggests that dye uptake continued for some time after the cessation of stimulation, as has been shown for vesicle recycling (cf. Heuser and Reese, 1973; Miller and Heuser, 1984).

We also studied the staining properties of mammalian muscles (Fig. 3). In rat lumbrical, soleus, and extensor digitorum longus muscle and mouse sternomastoid muscle, the intensity of motor nerve terminal staining depended upon transmitter release, as in frog muscle. To study the mechanism of the staining process in detail, we used the frog cutaneus pectoris preparation, and all other experiments reported here were performed with this preparation. 


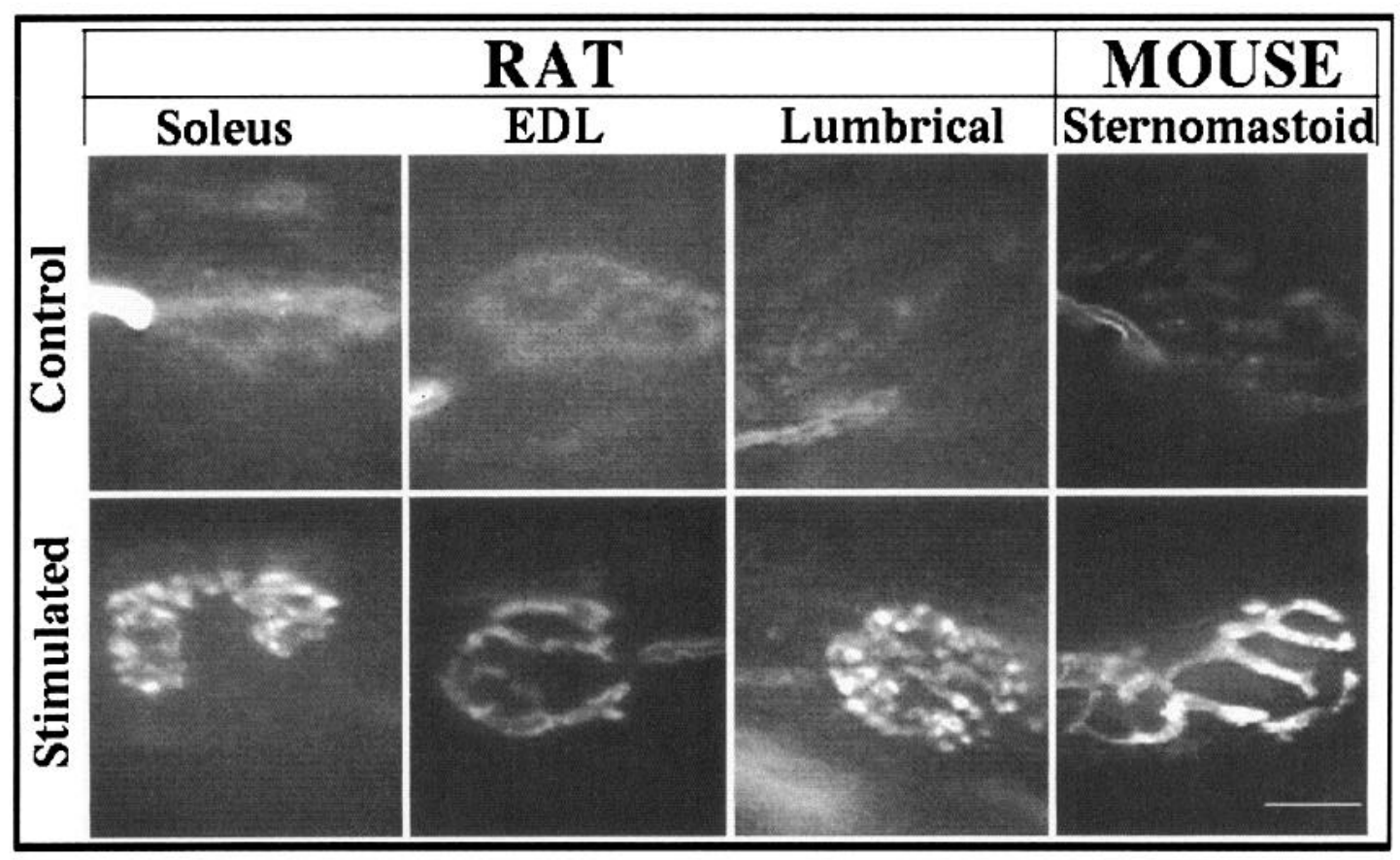

Figure 3. Rat and mouse motor nerve terminals stained in an activity-dependent fashion. Muscles were exposed to $2 \mu \mathrm{M}$ FM1-43 in normal Ringer's (Control) or in $60 \mathrm{~mm}\left[\mathrm{~K}^{+}\right]$-Ringer's (Stimulated) for $5 \mathrm{~min}$, washed, and imaged. Each image was acquired and processed to produce optimum contrast. Scale bar, $10 \mu \mathrm{m}$.

\section{Staining was not in muscle fibers}

Nerve terminals and Schwann cells can be dissociated from muscle fibers by treatment with collagenase and protease (Betz and Sakmann, 1973). When stained preparations were treated in this way, the fluorescent spots moved with the nerve terminal/ Schwann cell pairs (Fig. 4). Note also that the punctate nature of staining was preserved. Thus, the staining was not associated with muscle fibers.

\section{The final staining pattern emerged over several minutes}

Unlike the sulfonic acid dyes which stain snake motor terminals, the styryl dyes are only weakly fluorescent when dissolved in Ringer's solution (Grinvald et al., 1982). This enabled us to observe the onset of staining in the presence of the dye, as shown in Figure $5 A$. The top image was obtained before stimulation, about $1 \mathrm{~min}$ after adding the dye. The "railroad track" appearance of the nerve terminals is probably due to nonspecific dye uptake into the surface membranes of the cylindrical terminal and Schwann cells. Such staining, which grew progressively brighter regardless of nerve stimulation, could be washed out relatively easily. The lower panel in Figure $5 A$ was obtained after $15 \mathrm{sec}$ of electrical stimulation of the nerve $(60 \mathrm{~Hz} ; 9 \mu \mathrm{M}$ curare was present to block muscle contraction); note that the region between the railroad tracks brightened considerably. This type of brightening, which was not observed in unstimulated terminals, may reflect binding of the dye to synaptic vesicle membrane exposed to the extracellular fluid during exocytosis.

The initial activity-dependent brightening continued to change for several minutes, even if stimulation ended and dye was washed from the chamber (Fig. $5 B$ ). The panels in Figure $5 B$, which had background values subtracted individually, show a clear redistribution of dye after stimulation ended and dye washout began. While part of these changes may reflect enhanced contrast due to background dye washout, some also was clearly due to the gradual coalescence of the initial, rather diffuse dye pattern into patches.

\section{The final staining pattern was persistent}

While background staining of surface membranes of muscle fibers, Schwann cells, and myelin washed out relatively easily, the fluorescent patches were stable for many hours, even with continuous washing in dye-free medium. Terminals in Figure 6 were stained, imaged, washed for $16 \mathrm{hr}$, and reimaged. In the top panels (washed for $16 \mathrm{hr}$ at $4^{\circ} \mathrm{C}$ ), the size and position of nearly every patch remained fixed, and brightness dimmed by only $10-15 \%$. In terminals washed at room temperature (Fig. 6 , bottom panels), changes were more pronounced. The shape and position of some spots were altered, and brightness dimmed by $40-50 \%$. The changes in shape and position may reflect the beginning stages of nerve terminal degeneration due to the higher incubation temperature, and some of the loss of brightness was probably due to spontaneous transmitter release during the extended wash period.

\section{Fluorescent spots aligned with ACh receptors}

Figure 7 shows end plates from doubly stained muscles. In each panel, the pattern of postsynaptic AChRs is shown at the top (red). At the bottom of each panel, the nerve terminal, stained with FM1-43, is shown in green. Red and green images are aligned in the middle; regions of overlap appear yellow. There is a good correspondence between the two stains, although FM143 staining is more prominent at the lateral margins of the wide terminals.

Many of these results are consistent with the hypothesis that the dyes stain recycled synaptic vesicles and that each fluorescent spot marks a cluster of vesicles. However, the lateralization of patches in wide terminals (e.g., Fig. 7A) was puzzling, since 


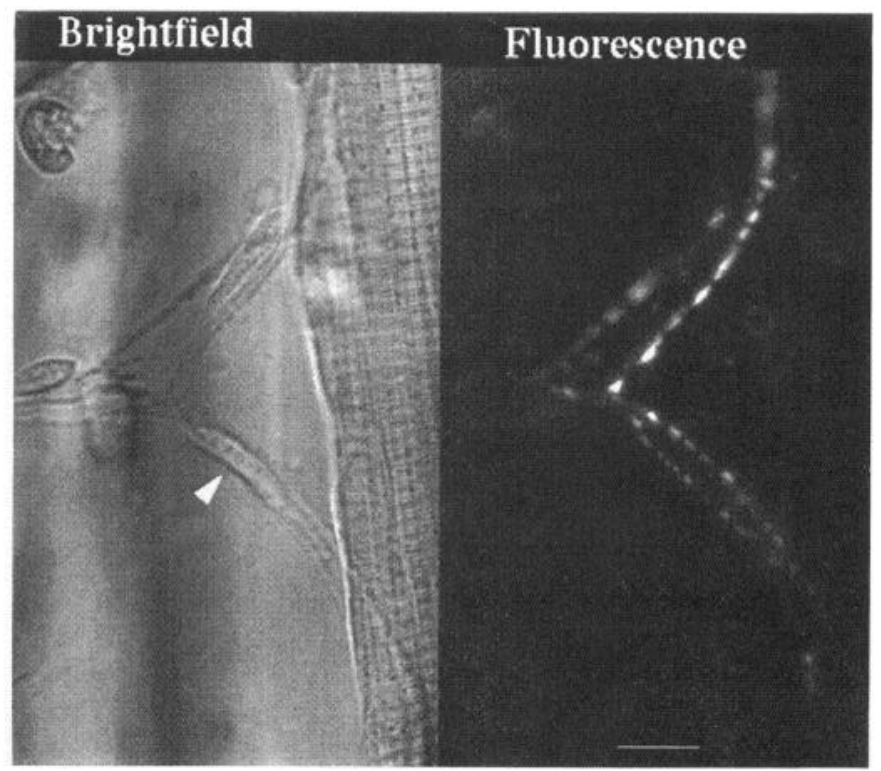

Figure 4. Fluorescent spots could be dissociated from muscle fibers. This preparation was stained with FM1-43 $\left(2 \mu \mathrm{M}\right.$ in $60 \mathrm{~mm}$ [K $\left.\mathrm{K}^{+}\right]$-Ringer's for $5 \mathrm{~min}$ ) and then incubated in $0.1 \mathrm{mg} / \mathrm{ml}$ collagenase for $15 \mathrm{~min}$, followed by $0.01 \mathrm{mg} / \mathrm{ml}$ protease for $30 \mathrm{~min}$, a procedure that dissociates pre- and postsynaptic cells from each other (left; arrowhead points to Schwann cell nucleus). The same field, viewed with fluorescence optics, showed that the fluorescence moved with the nerve terminals and Schwann cells (right). Scale bar, $10 \mu \mathrm{m}$.

published electron micrographs of frog motor nerve terminals almost always show synaptic vesicles in a single cluster. We therefore reexamined with the electron microscope the disposition of synaptic vesicles across the width of narrow and wide terminals, in both normal and stained preparations. Typical results are shown in Figure 8 (from a stained preparation). In the narrower terminals (left side), vesicles are indeed localized as central clusters (compare Fig. 7C), but in the wider terminals (right side), vesicles are spread rather nonuniformly across the width of the terminals. Thus, as was found with sulforhodamine staining of snake motor nerve terminals (Lichtman et al., 1989), the pattern of dye staining we have observed in frog motor nerve terminals is consistent with the distribution of synaptic vesicles in that preparation. In addition, unstained terminals were indistinguishable from stained ones, showing that the lateralization of staining was not due to disruption of normal cellular ultrastructure by the dye. While studies of identified terminals imaged with both fluorescence and electron microscopy will be necessary to quantify this feature in detail, the present observations show at least that, in wide terminals, synaptic vesicles can be distributed across the entire width in a pattern that could easily lead to lateralization of staining in fluorescence images.

\section{Nerve terminals failed to load with dye when stimulated to} exhaustion

In electron microscopic studies Ceccarelli et al. (1972, 1973) showed that stimulation of frog motor nerve terminals for $8 \mathrm{hr}$ at a low rate $(2 \mathrm{~Hz})$ exhausted the supply of synaptic vesicles. Thus, if the styryl dyes mark synaptic vesicles, a similar stimulation pattern during dye exposure should fail to produce the fluorescent spots characteristically observed after our usual dye-

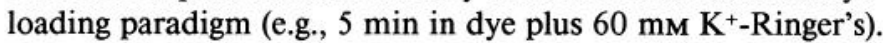
This was in fact observed, as shown in Figure 9. This preparation

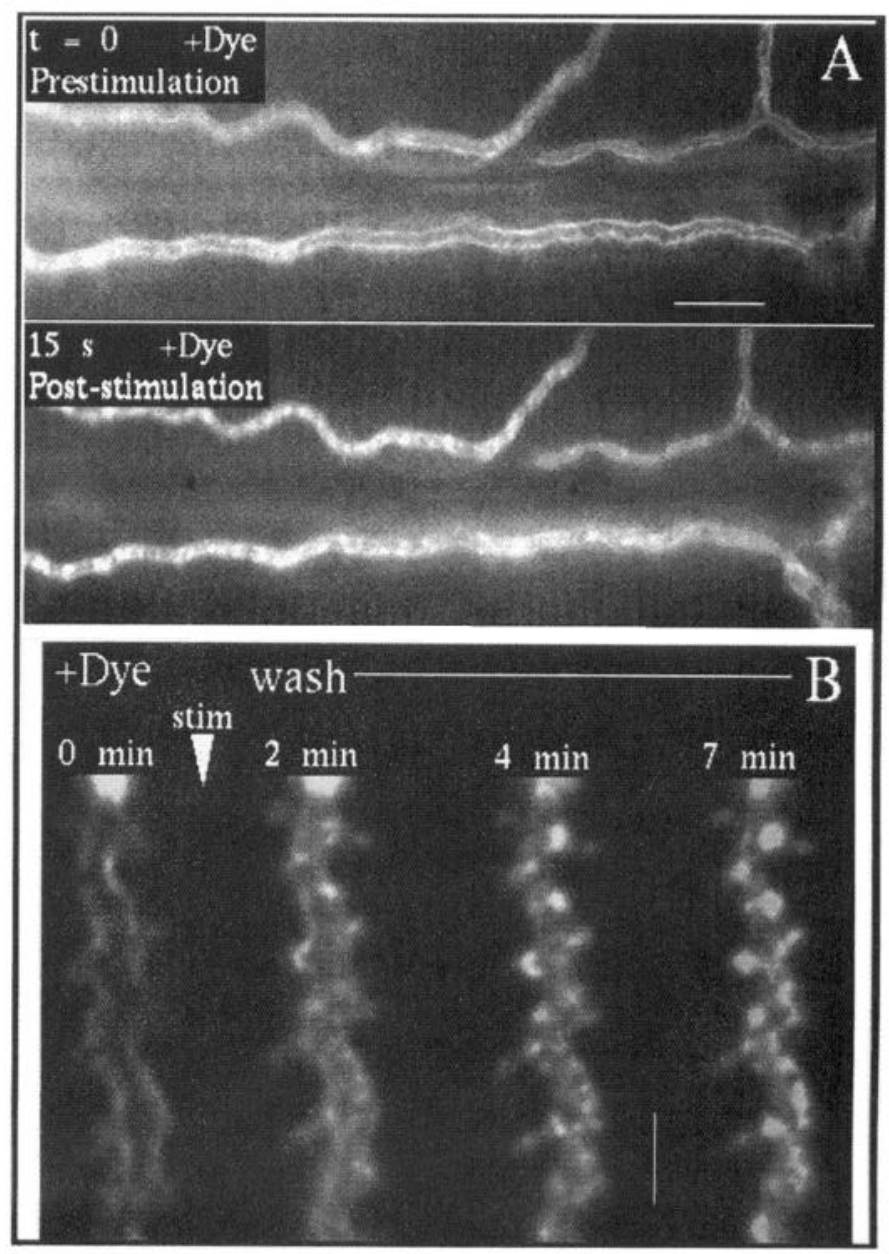

Figure 5. The time course of staining could be measured. $A$, In the top panel, FM1-43 (1 $\mu \mathrm{M})$ was present in the bathing solution and nonspecifically stained nerve terminals, giving a "railroad track" appearance. Such staining was easily washed out. After $15 \mathrm{sec}$ of nerve stimulation $(60 \mathrm{~Hz})$, the terminals brightened (lower panel). Both panels were imaged and processed identically. $B$, Illustrating the slow emergence of the final staining pattern, from a different preparation. The preparation was stimulated as in $A$ and then immediately washed with normal Ringer's. Images were obtained 2, 4, and $7 \mathrm{~min}$ after the nerve stimulation, with one or more washes in normal Ringer's applied between each image. Panels in $B$ were imaged identically but backgroundsubtracted individually. The slow emergence of fluorescent spots is evident. Scale bars: $A, 15 \mu \mathrm{m} ; B, 4 \mu \mathrm{m}$.

was stimulated at $1 \mathrm{~Hz}$ for $16 \mathrm{hr}$ in the continuous presence of $1 \mu \mathrm{M}$ FM1-43, washed, and imaged. The terminals are nearly devoid of the usual fluorescent spots. An especially clear example is shown in the middle panel, which shows a long stretch of terminal virtually devoid of fluorescent spots.

The images in Figure 9 are shown in color to illustrate a different observation, namely that the color of the dye varied according to the structure stained. Thus, myelin appeared green, while dye spots near Schwann cell nuclei (which probably mark endocytic vacuoles in Schwann cells) were orange. This illustrates the spectral lability of the dye; its color changed depending upon its microscopic environment.

\section{Other structures were stained}

Staining was not perfectly selective for motor nerve terminals. Three other structures also stained brightly. In each case, how- 
Figure 6. Fluorescent spots did not wash out easily. Muscles were stained in the usual way, imaged (Control), washed for $16 \mathrm{hr}$ at low temperature $\left(4^{\circ} \mathrm{C}\right)$ or room temperature $\left(20^{\circ} \mathrm{C}\right)$, and then reimaged (16 $\mathrm{hr}$ wash) with identical camera settings and processing. At $4^{\circ} \mathrm{C}$, few changes occurred during the wash period. At $20^{\circ} \mathrm{C}$, terminals dimmed by $40-50 \%$, and some spots became somewhat blurred. Scale bar, 8 $\mu \mathrm{m}$.

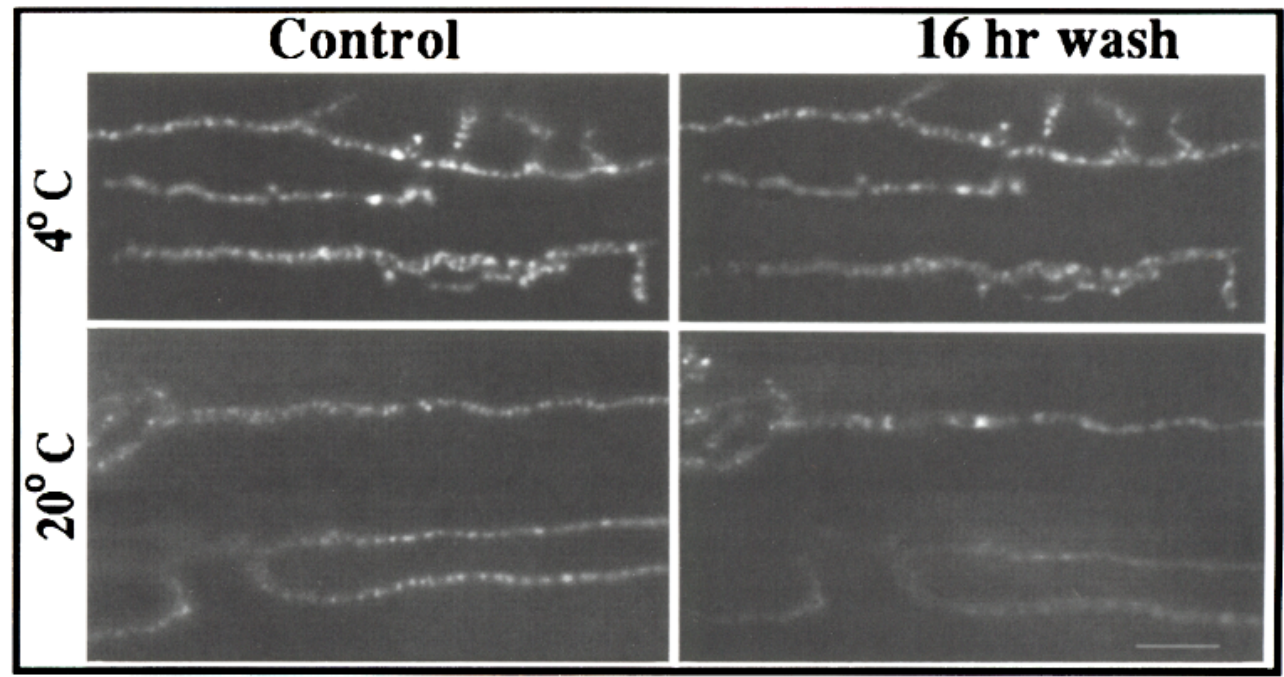

ever, staining intensity was independent of the amount of nerve stimulation. (1) Myelin. As noted above, myelin stained in an activity-independent fashion. This staining could be washed out, although after long exposure to a dye, the washout was slow. (2) Schwann cell vacuoles. These bright spots, which were generally smaller than those in nerve terminals, were seen in largest numbers in Schwann cell somata (e.g., Fig. 2, second panel), although some overlapped with nerve terminal spots, in which case it was not possible to determine whether they were in Schwann cells or in nerve terminals. These vacuoles had several characteristics that made them easy to identify. Their uptake did not depend on nerve activity, nor could they be destained (see below) by electrical nerve stimulation or elevated potassium concentration. In addition, such vacuoles often moved, and movements were relatively rapid and extensive (some moved more than $200 \mu \mathrm{m}$ in several minutes). Their
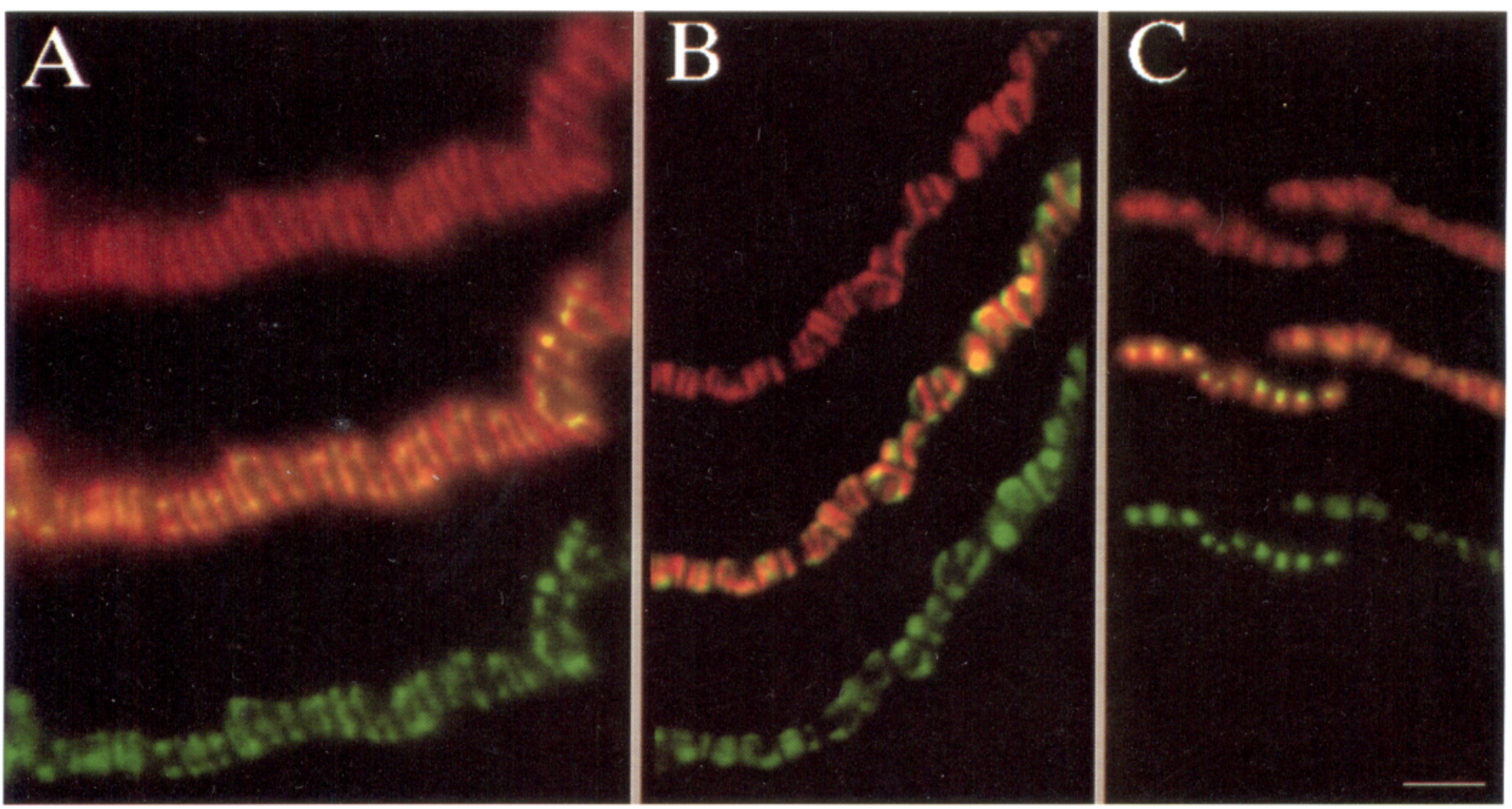

Figure 7. Fluorescent spots aligned with ACh receptors. Each of three end plates $(A-C)$ was stained with two dyes, rhodamine-conjugated $\alpha$-bungarotoxin (top, red) to stain ACh receptors, and FM1-43 (bottom, green) to stain nerve terminals, and viewed at $100 \times$. Each pair of images is superimposed in the middle (regions of red-green overlap are yellow). FM1-43 staining produced typical fluorescent spots which, particularly in the wider terminals $(A, B)$ are most prominent at the lateral margins of the terminals. Transverse bands of green dye connect the lateral patches and are aligned precisely with the red stripes of high receptor density, which mark positions of active zones in the terminals. Receptor stripes $(A)$ extend slightly beyond the FM1-43 spots, reflecting the curvature of the synaptic gutter viewed from above. 

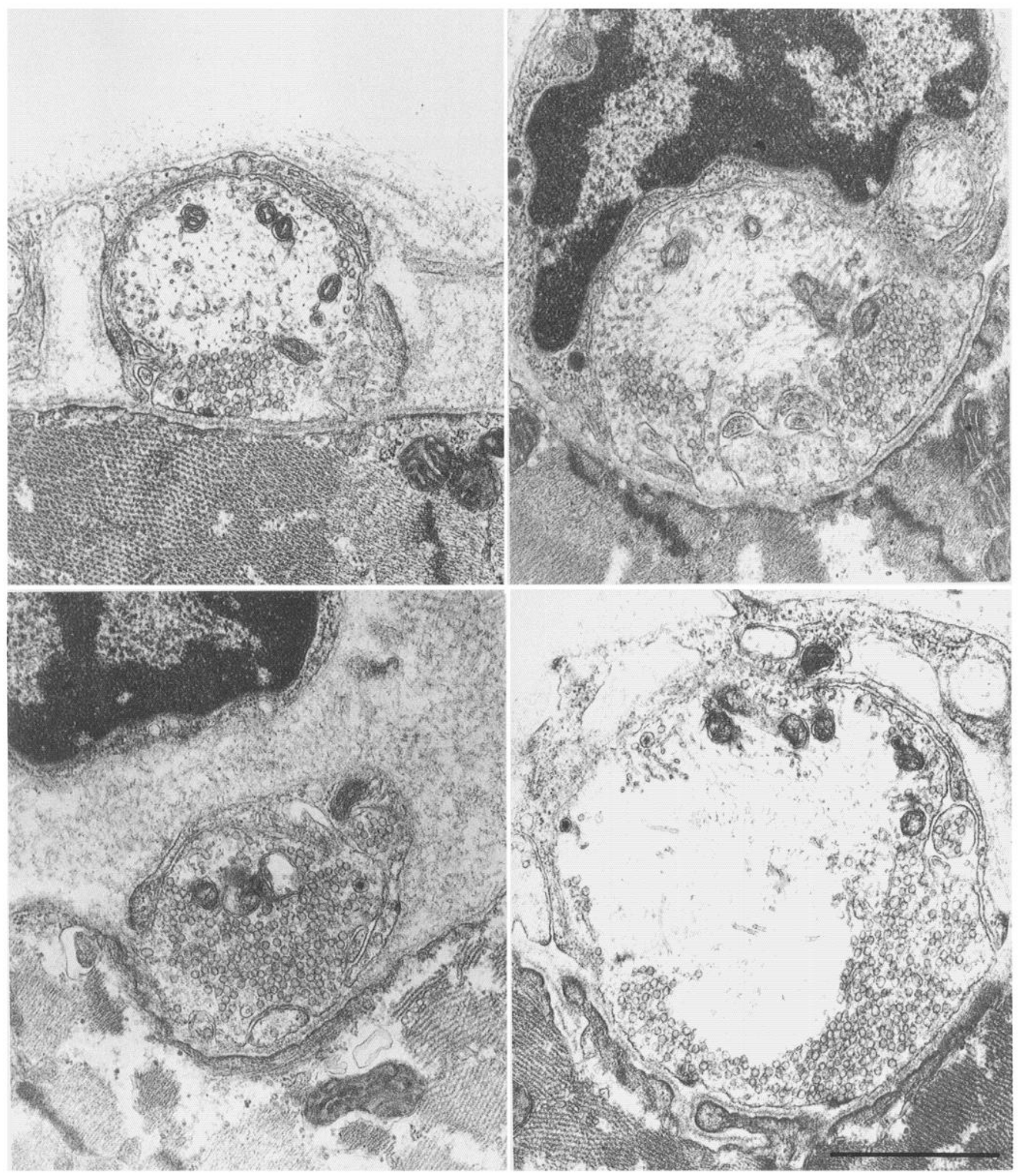

Figure 8. The distribution of synaptic vesicles was consistent with the shape of fluorescent spots. Typical electron micrographs of cross-sectioned terminals stained with FM1-43 show single, uniform vesicle clusters in narrow terminals (left panels), but less uniform clusters, often with lateral vesicle accumulations, in wide terminals (right panels). Scale bar, $1 \mu \mathrm{m}$.

movements did not appear to be affected by nerve stimulation. Their appearance always suggested that they were single structures, not clusters of vesicles (e.g., during movement, they did not break up). (3) Sensory nerve endings. Primary afferent (annulospiral) endings in muscle spindles stained intensely brightly.

\section{Destaining required transmitter release}

A key prediction of the hypothesis that the dyes label recycled synaptic vesicles is that the fluorescent spots should disappear when a stained preparation is stimulated in dye-free medium 
Figure 9. Terminals stimulated to exhaustion in the presence of dye lacked fluorescent spots. This preparation was stimulated electrically $(1 \mathrm{~Hz})$ for $16 \mathrm{hr}$ (a procedure that depletes synaptic vesicles) in the presence of $1 \mu \mathrm{M}$ FM1-43, washed, and imaged. Few fluorescent spots characteristic of normally loaded terminals are evident. In addition, the dye fluoresces with different colors, depending upon the structure stained. For example, myelin is green and Schwann cell vacuoles are orange. The preparation was photographed sequentially with two different emission bandpass filters (green and red). Images were processed individually for optimum contrast and superimposed. Scale bar, $10 \mu \mathrm{m}$.
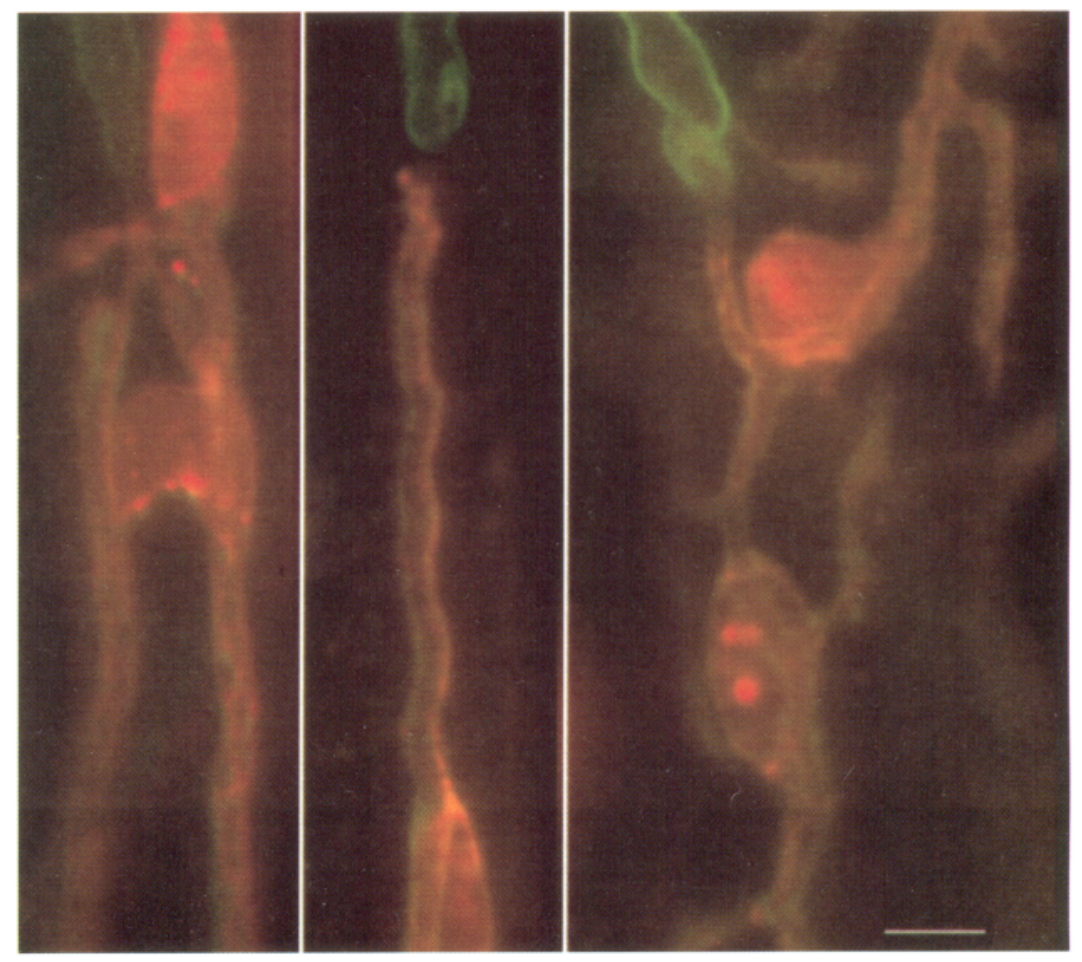

(cf. Heuser and Reese, 1973; Lichtman et al., 1985; Lichtman and Wilkinson, 1987). This was in fact observed (Fig. 10A). The patches faded gradually and uniformly (Fig. 10B). Typically, the initial rate of dye loss was linear, about $0.1-0.2 \%$ per stimulus at $5-10 \mathrm{~Hz}$. Fractional loss rate differed little between fluorescent patches belonging to the same terminal. The position and shape of each patch changed little during destaining, although sometimes patches viewed from the side appeared to dim first farthest from the synaptic membrane, as if the dye were moving toward the synaptic cleft. On rare occasions, a spot moved longitudinally within the terminal, appearing to be captured by a neighboring spot, which brightened as a result.

Destaining, like staining, was blocked when loaded terminals were stimulated in $\mathrm{Ca}^{2+}$-free Ringer's with added $\mathrm{Mg}^{2+}$ (Fig. $10 C)$. Thus, both staining and destaining depended upon transmitter release. We also found that activity-dependent destaining occurred in terminals after a $16 \mathrm{hr}$ wash, and in enzymatically dissociated terminals.

We attempted, without consistent success, to detect dye released by nerve terminals during destaining. Perfusates (both raw and extracted into various organic solvents to increase fluorescence intensity) were analyzed in a fluorimeter, but the signal intensity was too low to detect reliably. Neither was a bioassay (in which we attempted to stain freshly dissected nervemuscle preparations with perfusate from stained, stimulated preparations) reliably successful. We saw no significant retrograde dye movement within terminals or axons, nor did any combination of optical filters restore fluorescence to destained terminals. Thus, if the dye was not released from the terminals, it must have lost virtually all of its fluorescence across UV and visible spectra. We noted that in aqueous solution dye fluorescence was decreased by low $\mathrm{pH}$ ( $\mathrm{pH} 4-5)$, which, since synaptic vesicle contents are acidic, is in the opposite direction to explain the destaining. Thus, while not unequivocally proven that the dye is released to the extracellular medium during destaining, we will discuss our results based upon this assumption.

In general, fluorescent patches appeared along the entire length of each terminal, and while local variations in intensity were evident, we did not observe continuously graded differences along the length of terminals, either during staining or destaining, using either electrical or high- $\mathrm{K}^{+}$stimulation. A quantitative account of this is in preparation.

\section{Terminals could be repeatedly stained and destained}

The cycle of staining and destaining could be repeated, and fluorescent patches usually reappeared in the same positions (Fig. 11). This repeatability shows that the dye and method of loading are relatively nontoxic. We found, however, that extended exposure of loaded terminals to bright illumination, particularly during nerve activity, had toxic effects. The usual observation was that activity failed to induce destaining; the patches were rendered permanently bright by overillumination. This was not due to a failure of action potential conduction because focal overillumination of a short stretch of terminal did not block destaining (by electrical stimulation of the nerve) of distal portions of the terminal. We did not quantify this toxic effect in detail but found, for example, that no noticeable damage was produced by continuous illumination for $3 \mathrm{~min}$ with a $100 \mathrm{~W}$ $\mathrm{Hg}$ lamp through a $1 \%$ neutral density filter, $430-440 \mathrm{~nm}$ bandpass excitation filter and $40 \times$ water immersion objective $(0.75$ NA).

\section{Curare did not affect the rate of dye loss}

In addition to its well known postsynaptic blocking action, curare has been reported also to affect transmitter release (e.g., Magleby et al., 1981). Assuming that dye loss occurs during 


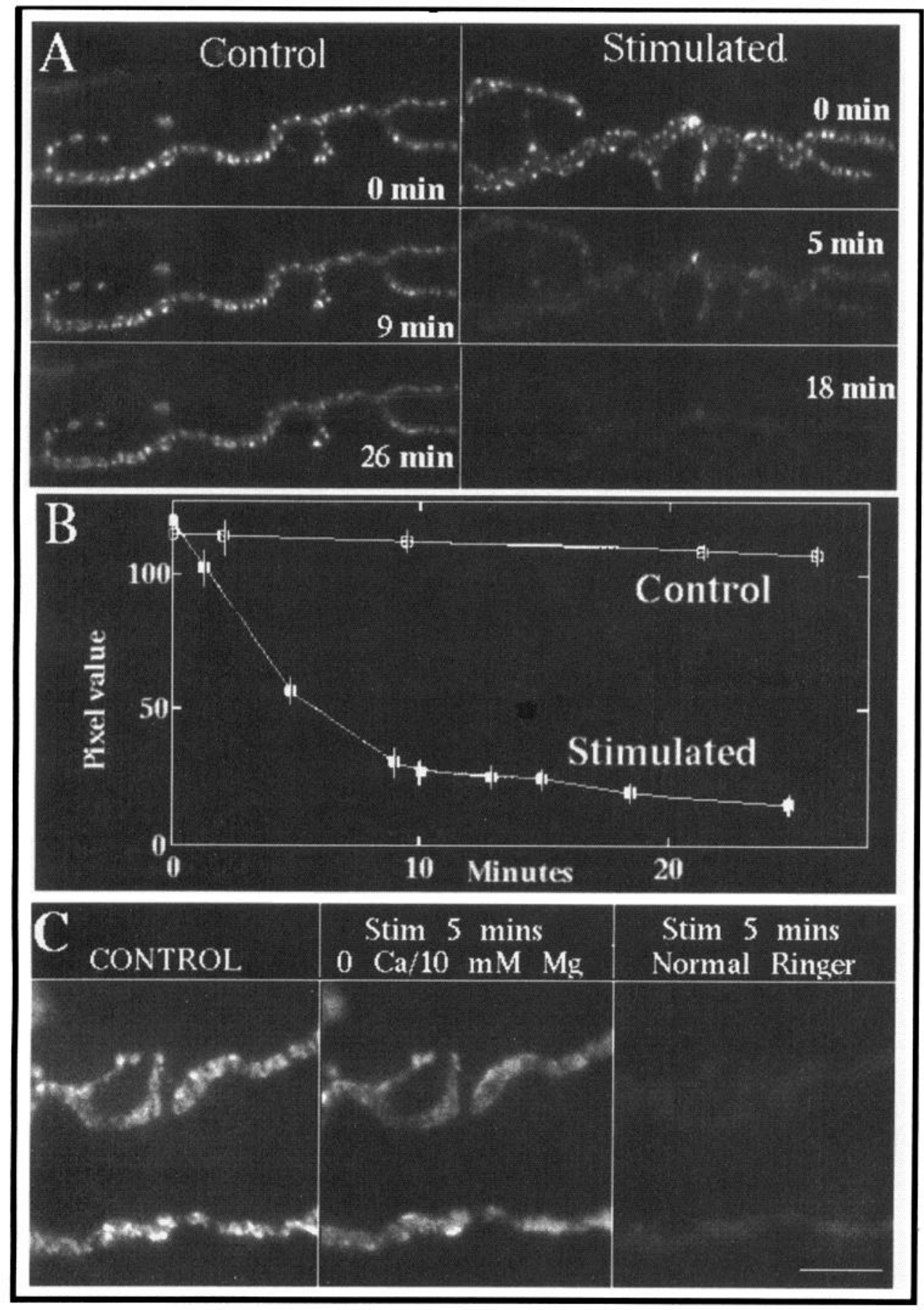

Figure 10. Destaining required transmitter release. $A$, Intramuscular nerve branches were cut near the middle of the muscle so that electrical stimulation activated terminals proximal (Stimulated), but not distal (Control) to the cut. All terminals in the muscle were then stained by exposure to $1 \mu \mathrm{M}$ FM143 in $60 \mathrm{~mm} \mathrm{~K}^{+}$-Ringer's for $5 \mathrm{~min}$, and the preparation was washed. The muscle nerve was stimulated at $5 \mathrm{~Hz}$ continuously, and images of control (left panels) and stimulated (right panels) terminals were obtained at the times indicated. Only the stimulated terminals destained. $B$, Each square marks the average brightness ( \pm 1 SEM) of eight specific fluorescent spots from the control and stimulated terminal. Background brightness was less than 10 at all times. $C$, In this muscle, all terminals were stained by exposure to $2.5 \mu \mathrm{M}$ FM1-43 in $60 \mathrm{mM} \mathrm{K}^{+}$-Ringer's for 2 min and then washed. Control images (left column) were then obtained. The preparation was then soaked in $\mathrm{Ca}^{2+}$. free Ringer's containing $10 \mathrm{mM} \mathrm{Mg}^{2+}$ for $40 \mathrm{~min}$, after which the terminals were again stimulated by exposure to $60 \mathrm{mM} \mathrm{K}^{+}$-Ringer's (with $0 \mathrm{mM} \mathrm{Ca}^{2+}$ and $10 \mathrm{mM} \mathrm{Mg}^{2+}$ ) for $5 \mathrm{~min}$ and washed. Then the same terminals were imaged again (middle column). Note that little destaining occurred when transmitter release was blocked by the $\mathrm{Ca}^{2+}$-free medium. Finally, the preparation was soaked in normal Ringer's for $30 \mathrm{~min}$ and then exposed to $60 \mathrm{mM} \mathrm{K}^{+}$-Ringer's for $5 \mathrm{~min}$, washed, and imaged again (right column). The last treatment caused substantial destaining. Scale bars: $A, 8 \mu \mathrm{m} ; C, 5 \mu \mathrm{m}$.

exocytsis of stained vesicles, then destaining experiments offer an independent, optical test of this hypothesis (Fig. 12). Thus, we measured the rate of destaining at single terminals in the presence and absence of curare. A preparation was incubated with unlabeled $\alpha$-bungarotoxin to block all AChRs, loaded with FM1-43, and then imaged during destaining ( $5 \mathrm{~Hz}$ continuous stimulation) in the presence of $9 \mu \mathrm{M}$ curare. The same preparation was then reloaded with dye, and after washing for $45 \mathrm{~min}$ in curare-free medium, the destaining and imaging procedures were repeated. The brightness of the same spots in each series was then measured, averaged, background-subtracted, and plotted (Fig. 12, graph). It is clear that curare had little effect on the rate of destaining.

\section{Discussion}

The results of the present experiments are consistent with the hypothesis that the styryl dyes RH414, RH795, and FM1-43 stain motor nerve terminals by marking recycled synaptic vesicles (Fig. 13). The mechanism of staining and its persistence can be explained most simply by a combination of high affinity for lipid membranes and an inability to penetrate membranes completely. The structure of the dyes (Fig. 13A) supports this conclusion. The hydrophobic tail would dissolve readily into the lipid membrane but would be prevented from passing right through by the highly charged, hydrophilic moiety at the opposite end of the molecule: Thus, the dyes seem to partition 
Figure 11. Terminals could be repeatedly stained and destained. This preparation was subjected to five cycles of loading and unloading (only the last three are shown) by exposure to $60 \mathrm{~mm}$ $\mathrm{K}^{+}$-Ringer's (5 min exposures, with 1 $\mu \mathrm{M}$ FM1-43 present during loading, but not unloading). Note that the bright spots generally reappeared in the same positions. Because there was a modest increase in background fluorescence with repeated dye exposures, the images were processed in pairs as indicated by the horizontal white lines. Scale bar, $6 \mu \mathrm{m}$.

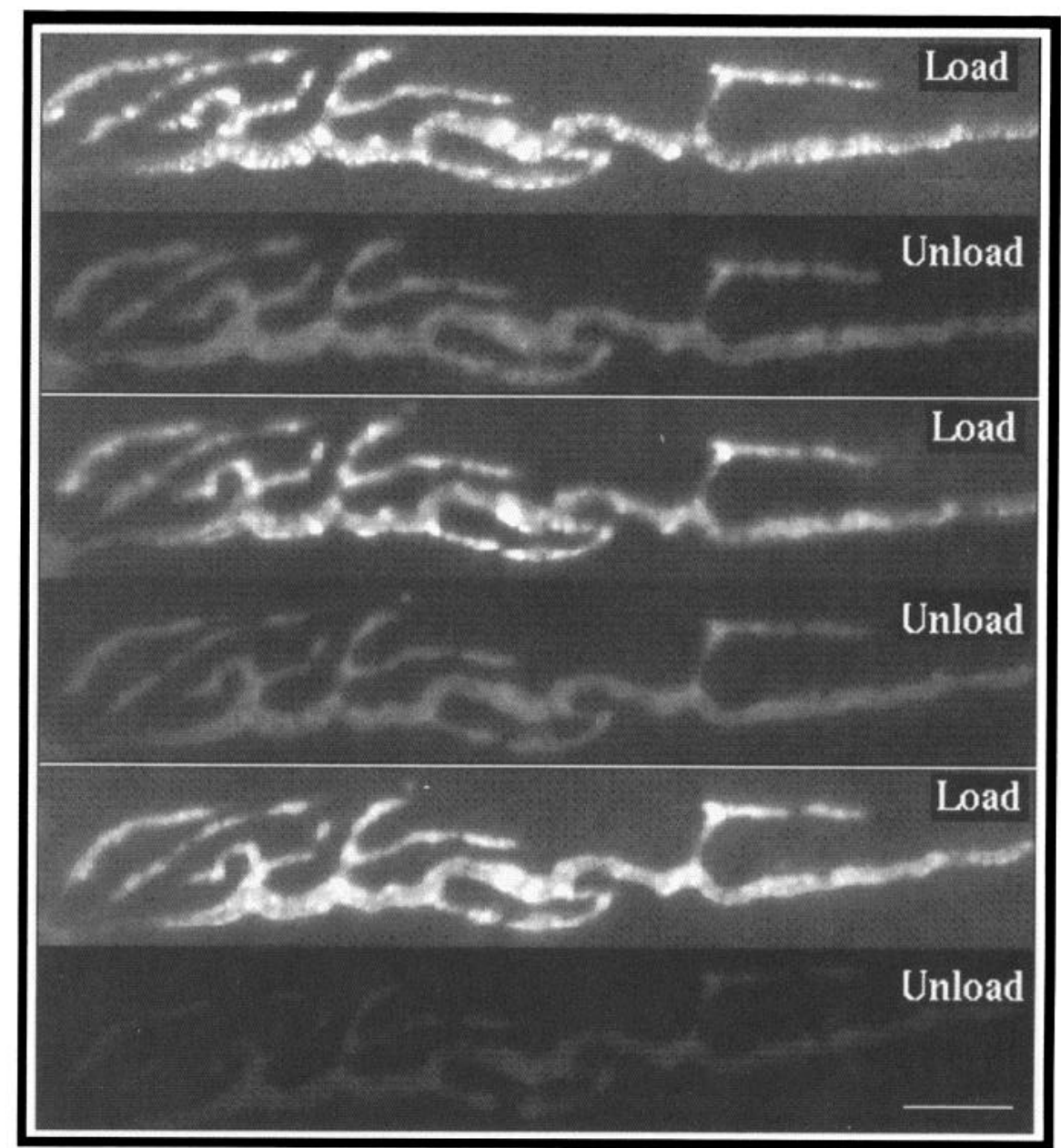

only into the outer leaflet of surface membranes (Fig. 13B). The increased fluorescence may result from simple partitioning of the dye into the membrane. However, the fact that the spectral properties of the dye can change according to its microscopic environment (Fig. 9) suggests additionally that the fluorescence of each dye molecule may increase in the lipid environment, as drawn in Figure $13 B$. During stimulation, the dye molecules become trapped in recycled vesicles during endocytosis (Fig. 13C) and can be subsequently lost with further stimulation (Fig. 13D). This staining mechanism differs from uptake of watersoluble markers in that the styryl dyes evidently mark vesicle membrane, and not vesicle lumen (although they are presumably present in both). The alignment of fluorescent patches with postsynaptic receptors, which mark the positions of active zones (Peper et al., 1974; Robitaille et al., 1990), is further evidence that the styryl dyes mark recycled vesicles. Furthermore, the pattern of fluorescent spots bears a striking resemblance to frog cutaneus pectoris nerve terminals stained with an antibody to synapsin I, a protein that is localized in regions of synaptic vesicle clusters (Valtorta et al., 1988a).

The fluorescent spots did not appear instantly after stimulation. Instead, immediately after a brief period of intense stimulation (e.g., $50 \mathrm{~Hz}$ for $15 \mathrm{sec}$, followed by dye washout) a rather diffuse brightening of the terminal was evident. Over the next few minutes, fluorescent spots gradually emerged. We did not study this process in detail because changes in background in- tensity during the washout period made it difficult to quantify accurately differences between images of spots obtained at different times. Brief, focal superfusion of dye during the stimulation period, rather than bath application of dye, would simplify this analysis. Thus, while the current results show clearly that dye distribution changed over a period of several minutes after stimulation, they do not distinguish between recycling routes involving clathrin-coated vesicles and intracellular cisternae (Heuser and Reese, 1973, 1981; reviewed in Heuser, 1989) or simple reversal of exocytosis (Ceccarelli et al., 1972; Torri-Tarelli et al., 1987; Valtorta et al., 1988b; reviewed in Valtorta et al., 1990).

Once the characteristic spots had formed, each was stable in position, shape, and intensity. A simple "cage" surrounding each spot, and thereby defining its boundaries, could explain these observations. While no such structure has been identified morphologically, in rapidly frozen, shallow-etched mouse cerebellar parallel fiber terminals, the cytoskeleton within vesicle clusters differs from the cytoskeleton in the immediately surrounding areas (Landis et al., 1988). Perhaps vesicles are caged by virtue of being incapable of partitioning into the adjacent cytoplasm, although at least some recycled vesicles would probably have to traverse this region to return to the vesicle cluster. An alternative, and more likely, mechanism for defining the boundaries of vesicle clusters involves the binding of individual vesicles to a meshwork of filaments localized in those regions 


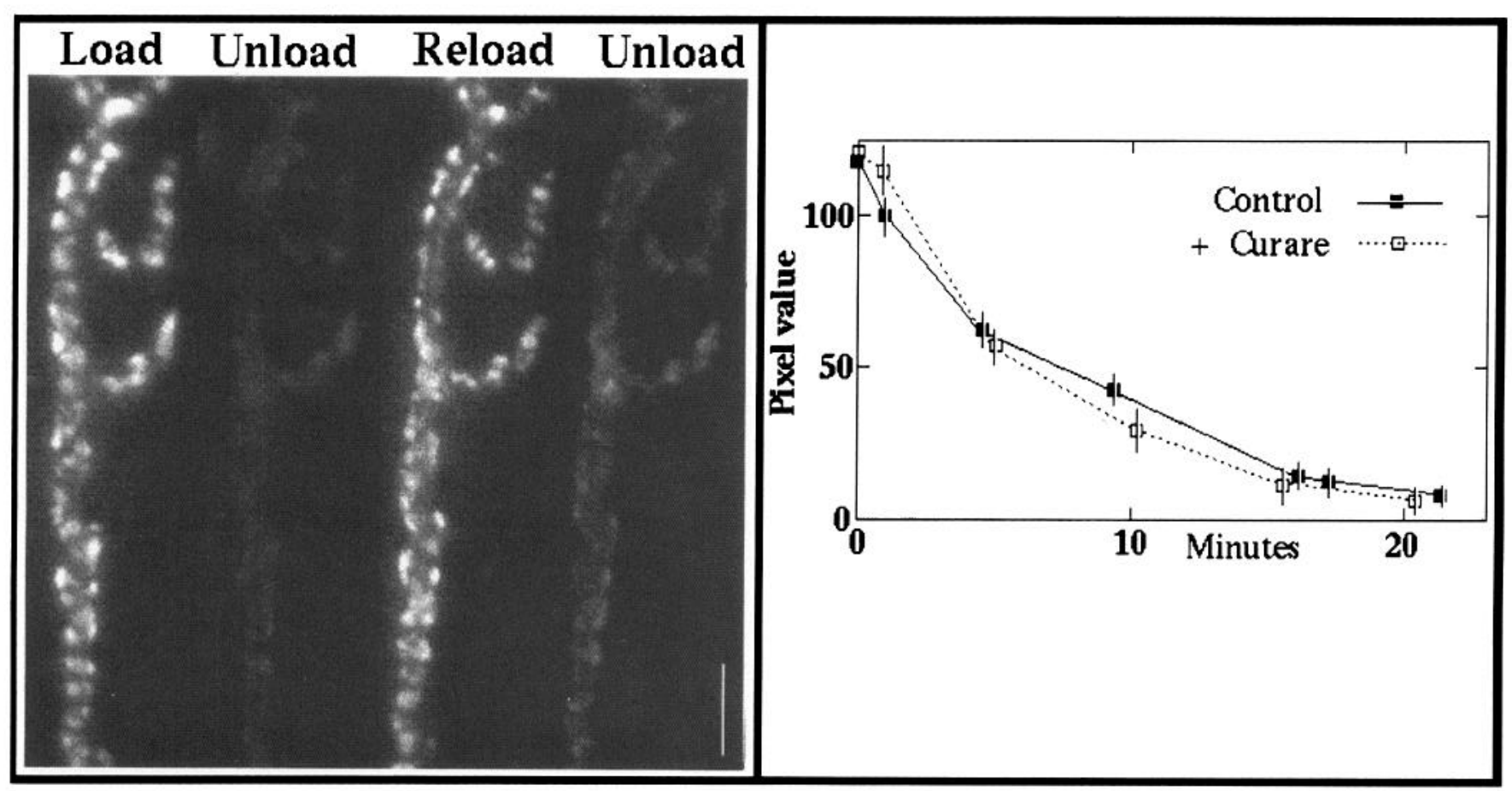

Figure 12. Curare did not significantly alter the rate of destaining. This preparation was stained in the usual way (Load) and imaged during destaining in the presence of $9 \mu \mathrm{M}$ curare; the last image in the series is shown (Unload). Images were acquired and processed identically and aligned, six spots were marked, and the average brightness of each spot in each image was measured. Averages ( \pm 1 SEM) for all six spots (after background subtraction) are plotted in the graph (open squares). The preparation was washed 60 min in curare-free medium, and the entire procedure was then repeated without curare (the same spots were measured), and plotted with solid squares. The rates of destaining were very similar. Scale bar, $5 \mu \mathrm{m}$.

(Landis et al., 1988; Hirokawa et al., 1989; Zimmermann et al., 1989). Some of these filaments are probably the filamentous protein synapsin I (Navone et al., 1984; Schiebler et al., 1986; Landis et al., 1988; Valtorta et al., 1988; Hirokawa et al., 1989; reviewed in Sudhof and Jahn, 1991).

If vesicles within a cluster are anchored at rest, how do they move toward the active zone during activity? In one model (Llinas et al., 1985, 1991), it was proposed that activity may initiate a sequence of events (calcium entry $\rightarrow$ activation of calmodulin kinase II $\rightarrow$ phosphorylation of synapsin I) that leads to the freeing of synaptic vesicles from their attachments to synapsin I. If the freed vesicles then move randomly by Brownian motion before docking at release sites at the active zone, adjacent fluorescent spots should have blurred and coalesced during destaining as freed vesicles spread out within the terminal. However, fluorescent spots seldom changed shape or position during destaining; they merely dimmed. Thus, vesicle movement is apparently restricted even during activity, which would require either a surrounding cage or a cytoskeletal translocator to move them toward the active zone. A hint of such a translocation system was observed in several terminals in which spots moved laterally within the terminal, appearing in time lapse movies to be swept toward and then captured by adjacent spots (which brightened as a result). Such movements must have involved the directed motion of hundreds or thousands of vesicles, which of course would require some kind of motor. At present, however, it is not clear if such a mechanism operates normally to move vesicles toward the active zone. Further studies of this type of vesicle movement could best be addressed by observing the terminals from the side, since the vesicles must then translocate across the field of view toward the active zone during destaining. Our preliminary analysis of such side views suggests that some spots destain first at the margin farthest from the active zone, as would be expected from a directed translocation process.

Destaining occurred at a rate of about $0.1 \%$ per stimulus at $10 \mathrm{~Hz}$. Assuming release of 300 quanta per stimulus per end plate, and assuming that dye loss from each exocytosed vesicle was complete, then about 300,000 vesicles in the terminal were stained by our usual loading procedure. This is within the usual range observed in ultrastructural studies of normal muscles (Ceccarelli et al., 1973; Heuser and Reese, 1973).

The dyes we used should be useful tools for some purposes, but they are not perfect. Their spectral properties can change, depending upon their microscopic environment (Fig. 9). Their emission spectra are quite broad, so that, while FM1-43 can be reliably distinguished from $\mathrm{RH} 414$ in the green, both are visible in the red (although the signal from FM1-43 is very faint). Neither do they stain motor nerve terminals with complete selectivity; myelin, primary afferent endings in muscle spindles, and endocytic vesicles in Schwann cells also stain. Each of these, however, is easily distinguishable from the stained vesicle clusters in motor nerve terminals. The dyes also can be toxic, especially when a stained terminal is overilluminated during nerve stimulation. Fortunately, reliable images can be obtained without signs of toxicity if proper precautions are taken.

Finally, in an attempt to illustrate the potential utility of the dyes for optical studies of transmitter release, we made preliminary measurements of the effects of curare on the rate of destaining. A terminal was destained twice in succession, once 


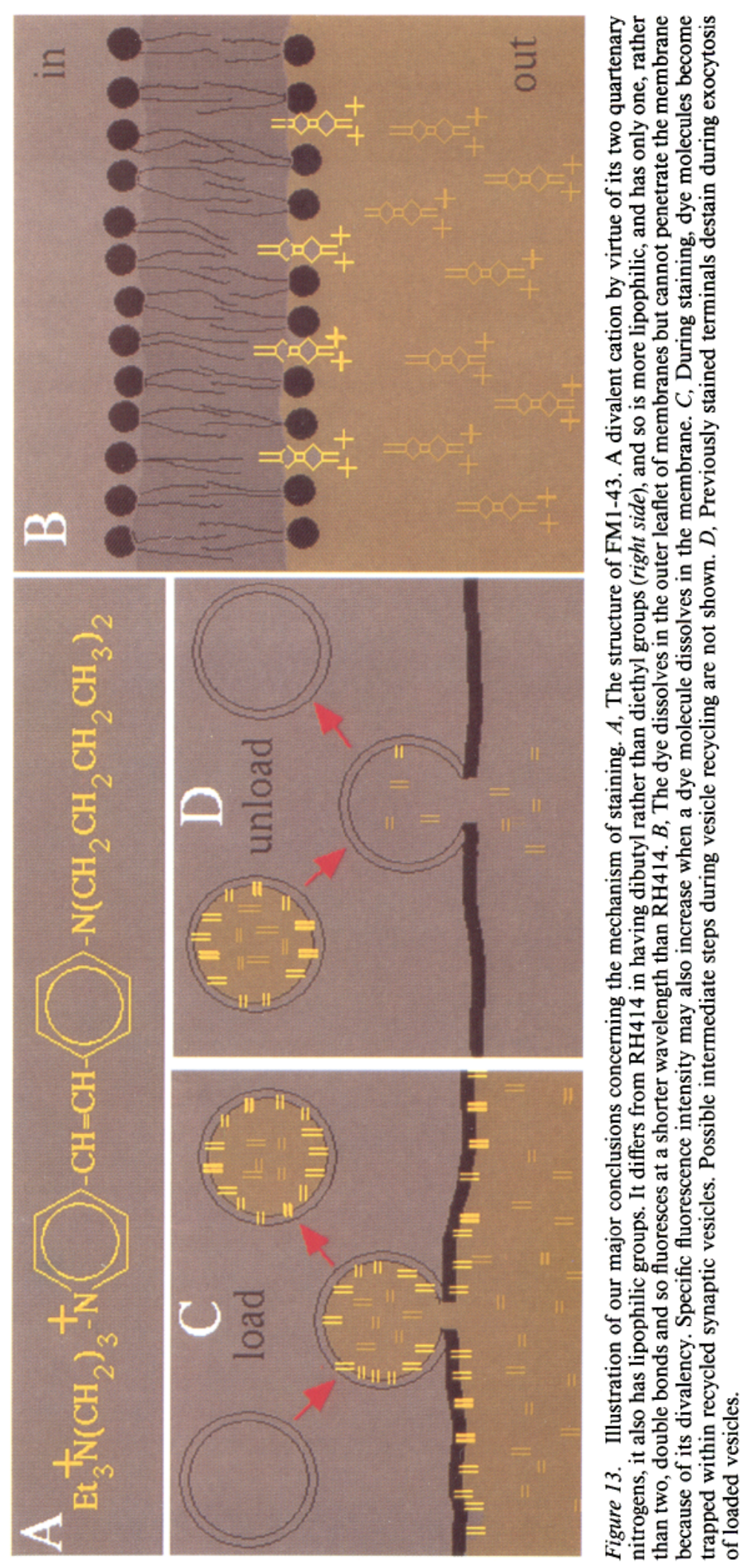


with and once without curare in the bathing solution. The destaining rates were very similar. Previous searches for presynaptic effects of curare on frog motor nerve terminals have been inconclusive, some showing no effect (e.g., Auerbach and Betz, 1971), others suggesting a presynaptic action (e.g., Magleby et al., 1981). These previous studies involved relatively short stimulus trains (less than $1 \mathrm{sec}$ in duration). The present measurements, extending over about $20 \mathrm{~min}$, give a more complete picture and, assuming that dye release provides an optical measure of transmitter release, offer tentative support for the conclusion that, compared to its well-established postsynaptic blocking action, curare has little or no effect on transmitter release during continuous stimulation in frog cutaneus pectoris motor nerve terminals.

In summary, with suitable precautions the styryl dyes should be useful tools for further cell biological studies of synaptic vesicle movements, for marking nerve terminals in an activitydependent fashion, and for optically monitoring transmitter release.

\section{References}

Auerbach A, Betz W (1971) Does curare affect transmitter release? J Physiol (Lond) 213:691-705.

Betz W, Sakmann B (1973) Effects of proteolytic enzymes on function and structure of frog neuromuscular junctions. J Physiol (Lond) 230: 673-688.

Betz WJ, Bewick GS (1990) Activity-dependent fluorescent staining of living rat motor nerve terminals with RH dyes. Soc Neurosci Abstr $16: 53$.

Ceccarelli B, Hurlbut WP, Mauro A (1972) Depletion of vesicles from frog neuromuscular junctions by prolonged tetanic stimulation. J Cell Biol 54:30-38.

Ceccarelli B, Hurlbut WP, Mauro A (1973) Turnover of transmitter and synaptic vesicles at the frog neuromuscular junction. J Cell Biol 57:499-524.

Graham RC, Karnovsky MJ (1966) The early stages of absorption of injected horseradish peroxidase in the proximal tubule of the mouse kidney; ultrastructural cytochemistry by a new technique. J Histochem Cytochem 14:291-302.

Grinvald A, Hildesheim R, Farber ID, Anglister L (1982) Improved fluorescent probes for the measurement of rapid changes in membrane potential. Biophys J 39:301-308

Grinvald A, Frostig RD, Lieke E, Hildesheim R (1988) Optical imaging of neuronal activity. Physiol Rev 68:1285-1367.

Heuser JE (1989) Review of electron microscopic evidence favouring vesicle exocytosis as the structural basis for quantal release during synaptic transmission. Q J Exp Physiol 74:1051-1069.

Heuser JE, Reese TS (1973) Evidence for recycling of synaptic vesicle membrane during transmitter release at the frog neuromuscular junction. J Cell Biol 57:315-344.

Heuser JE, Reese TS (1981) Structural changes after transmitter release at the frog neuromuscular junction. J Cell Biol 88:564-580.

Hirokawa N, Sobue K, Kanda K, Harada A, Yorifuji H (1989) The cytoskeletal architecture of the presynaptic terminal and molecular structure of synapsin I. J Cell Biol 108:111-126.

Holtzman E, Freeman AR, Kashner LA (1971) Stimulation-dependent alteration in peroxidase uptake at lobster neuromuscular junctions. Science 173:733-736.

Landis DMD, Hall AK, Weinstein LA, Reese TS (1988) The organization of cytoplasm at the presynaptic active zone of a central nervous system synapse. Neuron 1:201-209.

Lichtman JW, Wilkinson RS (1987) Properties of motor units in the transversus abdominis muscle of the garter snake. J Physiol (Lond) 393:355-374.

Lichtman JW, Wilkinson RS, Rich MM (1985) Multiple innervation of tonic endplates revealed by activity-dependent uptake of fluorescent probes. Nature 314:357-359.

Lichtman JW, Sunderland WJ, Wilkinson RS (1989) High-resolution imaging of synaptic structure with a simple confocal microscope. New Biologist 1:75-82.

Llinas R, McGuinness TL, Leonard CS, Sugimori M, Greengard P (1985) Intraterminal injection of synapsin I or calcium/calmodulindependent protein kinase II alters neurotransmitter release at the squid giant synapse. Proc Natl Acad Sci USA 82:3035-3039.

Llinas R, Gruner JA, Sugimori M, McGuinness TL, Greengard P (1991) Regulation by synapsin $\mathrm{I}$ and $\mathrm{Ca}^{2+}$-calmodulin-dependent protein kinase II of transmitter release in squid giant synapse. J Physiol (Lond) 436:257-282.

Magleby KL, Pallotta BS, Terrar DA (1981) The effect of (+)-tubocurarine on neuromuscular transmission during repetitive stimulation in the rat mouse and frog. J Physiol (Lond) 312:97-113.

Miller TM, Heuser JE (1984) Endocytosis of synaptic vesicle membrane at the frog neuromuscular junction. J Cell Biol 98:685-698.

Navone F, Greengard P, DeCamilli P (1984) Synapsin I in nerve terminals: selective association with small synaptic vesicles. Science 226:1209-1211.

Peper K, Dreyer F, Sandri C, Akert K, Moor H (1974) Structure and ultrastructure of the frog motor end plate. Cell Tissue Res 149:437455.

Robitaille R, Adler EM, Charlton MP (1990) Strategic location of calcium channels at transmitter release sites of frog neuromuscular synapses. Neuron 5:773-779.

Schiebler W, Jahn R, Doucet JP, Rothlein J, Greengard P (1986) Characterization of synapsin I binding to small synaptic vesicles. J Biol Chem 261:8383-8390.

Sudhof TC, Jahn R (1991) Proteins of synaptic vesicles involved in exocytosis and membrane recycling. Neuron 6:665-677.

Torri-Tarelli F, Haimann C, Ceccarelli B (1987) Coated vesicles and pits during enhanced quantal release of acetylcholine at the neuromuscular junction. J Neurocytol 16:205-214.

Valtorta F, Villa A, Jahn JE, DeCamilli P, Greengard P, Ceccarelli B (1988a) Localization of synapsin I at the frog neuromuscular junction. Neuroscience 24:593-603.

Valtorta F, Jahn R, Fesce R, Greengard P, Ceccarelli B (1988b) Synaptophysin (p38) at the frog neuromuscular junction: its incorporation into the axolemma and recycling after intense quantal secretion. J Cell Biol 107:2717-2727.

Valtorta F, Fesce R, Grohovaz F, Haimann C, Hurlbut WP, Iezzi N, Torri Tarelli F, Villa A, Ceccarelli B (1990) Neurotransmitter release and synaptic vesicle recycling. Neuroscience $35: 477-489$.

Zimmermann $H$ (1979) Vesicle recycling and transmitter release. Neuroscience 4:1773-1804.

Zimmermann H, Volknandt W, Henkel A, Bonzelius F, Janetzko A, Kanaseki T (1989) The synaptic vesicle membrane: origin axonal distribution protein components exocytosis and recycling. Cell Biol Int Rep 13:993-1006. 